\title{
Challenges and successes in implementing active learning laboratory experiments for an undergraduate analytical chemistry course
}

\author{
Anna G. Cavinato ${ }^{1}$ \\ Published online: 9 December 2016
}

(C) Springer-Verlag Berlin Heidelberg 2016

\section{Introduction}

Recently, the White House Office of Science and Technology Policy issued a national call to action to improve Science, Technology, Engineering, and Mathematics (STEM) education through the use of active learning [1]. The call encourages educators at all levels to use active learning strategies to better prepare students to meet the nation's growing demand for jobs in science and technology fields. In an active learning approach, rather than watching or listening to the instructor or following cookbook-like lab procedures, students work collaboratively in small groups on carefully crafted classroom or laboratory activities. The activities are designed to engage students in higherorder cognitive processes when presented with new concepts or open-ended questions, to help them develop connections, and ultimately to construct their own knowledge [2]. A rich literature exists advocating for changes in lecture and lab format [3-7], and studies show that student-centered methods of instruction such as small-group collaborative learning, inquiry, and projectbased activities appear more effective in improving students' performance and retention [8, 9]. Furthermore, traditional laboratory experiments do not lead to desired learning outcomes such as problem-solving skills, critical thinking, the ability to design and implement experiments, and apply knowledge in real-world settings $[2,10]$.

Educators associated with the Analytical Sciences Digital Library (ASDL) [11] have been long-term proponents of active learning curriculum and, over the years, have developed an online collection of materials in the area of chemical analysis

Anna G. Cavinato

acavinat@eou.edu

1 Department of Chemistry and Biochemistry, Eastern Oregon University, One University Blvd., La Grande, OR 97850, USA and instrumentation, including in class and laboratory activities and contextual modules [12].

In an effort to involve students in lab experiences that engage them in the analytical process, I have developed a curriculum based on open-ended research projects usually dictated by a community partner. The approach is intended to engage students in all aspects of the process, from researching the experimental procedures that best suit the research question, to assembling the required materials and supplies, executing the experiments and analyzing, interpreting, and reporting the data.

The activities described are implemented in an Analytical Chemistry Laboratory course. This is an upper division course that students typically take in their second or third year of college. Although students have been introduced to some techniques such as titrations or spectroscopic analysis (UVVis and atomic absorption spectroscopy) in the general chemistry laboratory, this course represents the first exposure to more rigorous analytical work. The class meets twice a week for a 3-h block lab during the course of 10 weeks and has a typical enrollment of 20 students. Experiments range from traditional wet methods such as determining water hardness by EDTA titration to instrument-based techniques, including chromatography, spectroscopy, and electrochemistry. Emphasis is placed on comparing results obtained on the same samples using different techniques. Broad research questions are pursued in collaboration with community partners who are interested in the outcome of the analyses but are also invested in providing real-world learning opportunities to students.

\section{Specific examples of laboratory projects}

Over ten different projects have been pursued during the last 10 years in collaboration with community partners. Some recent examples include: 
- Grande Ronde Model Watershed/Native American Tribes - What water conditions provide a suitable habitat for the Columbia spotted frogs?

- US Forest Service - Is the water quality of a local stream good enough to support salmonids population?

- Oregon Department of Fish and Wildlife - Is the city wastewater treatment plant affecting the water quality of a protected marsh, home to endangered species?

In designing the lab activities, I kept in mind the guidelines on what constitutes active learning in a laboratory environment. These guidelines, developed by a team of ASDL educators, are outlined in Table 1.

Active learning strategies vary during the course of the term starting from guided inquiry and progressing to an independent group project. A breakdown of lab activities conducted on a weekly basis is presented in Table 2 .

At the beginning of the term, students are introduced to the research question by a scientist representing the community partner. The scientist outlines the goals of the project in the context of a broader mandate specific to that organization. For example, in one instance, a field biologist from the US Forest Service described efforts in understanding the impact of different grazing practices on salmon restoration. The project revolved around a creek located in a US Forest Service experimental research station along which different grazing practices were implemented. The scientist identified specific sites and helped students design a sampling plan to monitor water quality in the stream. Other examples of themes and case studies used to provide a real-world context for project-

Table 1 Guidelines for developing active learning laboratory activities

The activity begins with an open-ended question or problem and is designed so that students work in teams

The instructor may or may not know the outcome of the problem in advance

Students must use prior knowledge and/or consult the literature to address the question or problem

Students must use critical thinking skills to develop a hypothesis and/or devise a procedure to solve the problem following appropriate aspects of laboratory safety

Students will either carry out the procedure by observing and collecting information or be provided data when they have determined the correct measurements to make

When experiments are performed, students must make decisions about their design and execution, taking into account aspects of safety

Students will use appropriate methods to validate their measurements

Students will process the information that is collected or provided (e.g., describe, tabulate, summarize, calculate)

Students will draw conclusions from the information that is collected or provided and support those conclusions

Students will usually report on their findings in written and/or oral form
Table 2 Example of project-based analytical chemistry lab schedule

\begin{tabular}{ll} 
Week 1 & Introduction to analytical methods - sampling \\
Week 2 & Meet community partner - develop sampling plan \\
Week 3 & Field trip - collection of water samples \\
Week 4 & Determination of $\mathrm{Ca}^{2+}$ and $\mathrm{Mg}^{2+}$ by EDTA and AAS \\
Week 5 & Determination of $\mathrm{Ca}^{2+}$ and $\mathrm{Mg}^{2+}$ by EDTA and AAS \\
Week 6 & Determination of nitrate content by ISE and IC \\
Week 7 & Determination of nitrate content by ISE and IC \\
Week 8 & Lab project \\
Week 9 & Lab project \\
Week 10 & Lab project - report to community partner \\
\hline
\end{tabular}

$A A S$ atomic absorption spectroscopy, ISE ion-selective electrode, $I C$ ion chromatography

based analytical labs can be found on the ASDL Active Learning Curriculum Materials [12].

After students are introduced to the project, they are provided with maps of the area so that they can devise a sampling plan to be implemented during a field trip to the area under study. During the first week of the term, they learn about fundamental concepts on how to design a sampling plan and obtain and prepare samples for analysis. An excellent discussion on sampling and sample preparation can be found in Chap. 7 of the free textbook Analytical Chemistry 2.0 by David Harvey [13]. Students are then divided into small groups and given the task to design a specific sampling plan that includes the number of sites to sample, the amount of water to collect, and how to treat samples for long-term storage. During the field trip, sites are identified by GPS coordinates and mapped for reporting purposes.

In this approach to teaching labs, the outcome of the analyses is not known. Some of the general analysis protocols are provided, although students still have to figure out details of the procedure. For the final project, students must research the literature and devise a procedure to carry out the analyses.

Several analyses are conducted on-site using simple field kits and probes while more involved analyses are conducted back in the lab on water samples collected at the different sampling sites and brought back for longer-term storage. Table 3 summarizes typical experiments conducted on-site and off-site. Part of the goal in conducting such measurements using different tools is for students to develop a sense of their appropriateness based on different sensitivities and detection limits. A discussion of the different analytical approaches and details of experimental procedures can be found in the contextual module End Creek: Spotted Frogs and Aquatic Snails in Wetlands - A Water Quality Investigation published on the ASDL site [12].

In the second part of the term students transition to an independent lab project which, in this particular example, dealt with the determination of chlorinated pesticides by 
Table 3 Example of measurements conducted on-site during the field trip and off-site (in lab) on water collected at the specific sampling sites

\begin{tabular}{ll}
\hline On-site measurements & Off-site measurements \\
\hline Temperature & $\begin{array}{c}\text { Metals (Ca, Mg) (EDTA titration and } \\
\text { atomic absorption spectroscopy) }\end{array}$ \\
$\begin{array}{l}\text { pH (Hach kit and solid state } \\
\text { sensor) }\end{array}$ & $\begin{array}{c}\text { Nitrates (ion-selective electrode and ion } \\
\text { chromatography) }\end{array}$ \\
$\begin{array}{l}\text { Dissolved oxygen (Hach kit } \\
\text { and electrochemical sensor) }\end{array}$ & Pesticides by GC-MS \\
Conductivity & \\
Nitrates (Hach kit) & \\
Phosphates (Hach kit) & \\
Chlorides (Hach kit) & \\
\hline
\end{tabular}

gas chromatography-mass spectrometry (GC-MS). Each group prepares a proposal in where, on the basis of a review of published methods, they choose the appropriate method of analysis, develop a list of lab supplies, and outline, to the best of their abilities, the experimental protocol. An excellent resource that provides activities related to the use of GC-MS for the determination of chlorinated pesticides in water is the contextual module Environmental Analysis - Lake Nakuru Flamingos: Pesticides [12]. After being introduced to GC-MS, students are directed to research EPA method 525.2 and provide details on the type of chromatographic and solid-phase extraction (SPE) columns best suited for analysis. Quantitation may be challenging depending on the course level but qualitative analysis can be reasonably implemented using chlorinated pesticides standards.

\section{Learning outcomes and assessment}

Several learning outcomes can be achieved by using active learning, open-ended activities in an analytical chemistry laboratory course. Basing the lab on an authentic research experience challenges students to operate more independently and seek strategies and solutions that help them develop analytical skills. Students are involved with all aspects of the analytical process from developing a sampling strategy to devising and carrying out the experimental procedure based on published methodologies and analyzing and interpreting the results. Other learning outcomes address communication skills such as learning to communicate to different audiences, and understanding regulatory policies. Overall, working with a community partner help students become more aware of local issues and use scientific knowledge to benefit the community.

Multiple tools are used in the course to assess the impact of active learning laboratory experiments on students' learning. These include individual laboratory reports structured as research papers, a final presentation to the community partner, and a reflection paper on the overall experience. Pre- and post-test assessments can also be administered. For example, Fig. 1 compares the percentage of correct answers for a 15 multiple choice question quiz designed to assess the impact of lab activities on students' understanding of sampling, sample preparation for analysis, preparation of standards, dilutions, and calibration curves. This assessment, conducted over the past 5 years $(N=100)$, indicates increase in student understanding of fundamental concepts related to sampling, purpose of standards, and instrument calibration. A representative sample of questions is reported in Table 4.

The reflection paper provides a chance for students to comment on their lab experience both in terms of their ability to learn new concepts and apply them to the solution of a new problem. Some common positive comments as well as concerns typically emerge. Students highly praise the opportunity to conduct analyses in the field. By being exposed to all aspects of an analysis, from collecting and preserving samples, to choosing an appropriate method of analysis, gathering reagents and supplies, and conducting the measurements, they feel these activities reinforce their knowledge of concepts discussed in class. Many students appreciate the opportunity to collaborate with a community partner. The collaboration brings a sense of purpose and ownership to the analyses. It helps students become more aware of concerns related to local environmental issues and the fact that there are federal and local regulations to safeguard the environment. However, students also voice concerns about the way the lab is conducted. Some find the project overwhelming and lament limited time to perform the analyses. They dislike not having a developed procedure and wish that detailed instructions had been provided. The final report to the community partner is also considered by a few as overwhelming while others feel uncomfortable reporting results that they do not deem accurate because of limited time to repeat the analyses.

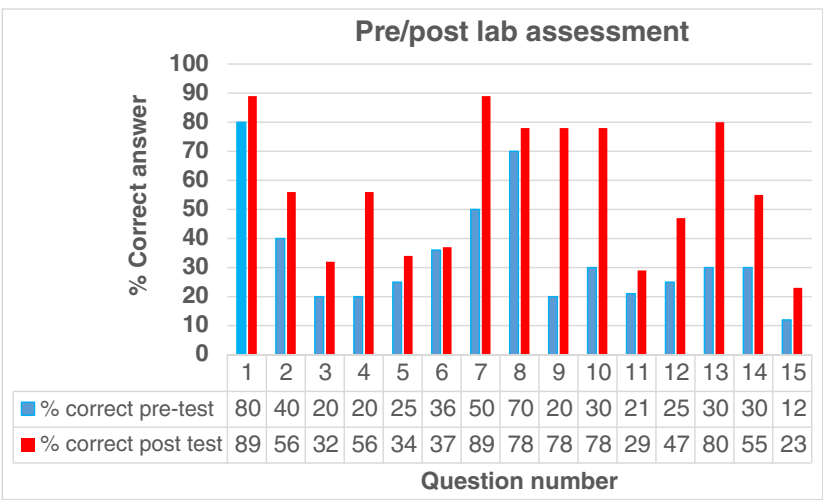

Fig. 1 Results of a pre- and post-lab assessment administered over five different terms $(N=100)$ 
Table 4 Example of multiple choice questions used in the assessment results presented in Fig. 1
1. What factors should be considered when designing a sampling plan?
A. Information about the process or area of concern
B. Data quality information
C. Constraints
D. All the above factors need to be considered

2. Suppose you have knowledge of a point source of pollution. What type of sampling design would be more appropriate to use?
A. Random
B. Systematic
C. Stratified
D. Judgmental

3. What is the significance of analyzing for dissolved oxygen in a stream?

A. To determine the amount of oxygen available to aquatic life

B. To determine the oxidizing power of river water

C. To determine presence of fertilizers in the water

D. To measure the water flow in the stream

4. What is the purpose of a standard in chemical analysis?

A. To indicate when the analysis is complete

B. To provide a known concentration of the analyte

C. To provide an estimate of the error in the analysis

D. To provide the average value of the concentration of the analyte

5. What instrumental technique would you use to detect the presence of calcium and magnesium in water?
A. Atomic absorption spectroscopy
B. Gas chromatography-mass spectrometry
C. Potentiometer
D. UV/Visible spectrophotometry

6. A 500.0-mL sample of river water is found to contain $0.075 \mathrm{mg}$ of iron. What is the concentration of copper in ppm?
A. $1.5 \times 10^{-4} \mathrm{ppm}$
B. $0.15 \mathrm{ppm}$
C. $6.7 \mathrm{ppm}$
D. $6.7 \times 10^{3} \mathrm{ppm}$

7. What is a calibration curve?

A. A bend in the path of a stream

B. The response of the flow of water to variations in aquatic life

C. The change of the analyte in the water stream

D. The response of an instrument to variation in the concentration of the analyte

8. A calibration curve for the spectrophotometric determination of phosphate in water is prepared by reacting each standard solution with a reagent to produce a blue color. The calibration equation is as follows: $A=0.205 x+0.004$ where $A=$ absorbance, $x=$ concentration of phosphorous. What is the concentration of phosphorous if the absorbance of an unknown water sample is 0.625 ?
A. $3.03 \mathrm{ppm}$
B. $3.05 \mathrm{ppm}$
C. $105 \mathrm{ppm}$
D. Not enough information provided

9. What is the "limit of detection" of an analysis?

A. The instrument response change with changes in concentration of the analyte

B. The limit in the operator's ability to read the response of a given instrument

C. The minimum concentration level of the analyte that can be detected

D. The minimum amount of reagent that needs to be added to perform the analysis 


\section{Challenges and successes}

There are a number of inherent challenges associated with the implementation of active learning lab activities, although many positive outcomes can be identified. Table 5 outlines challenges and successes that this author has observed over the course of several years and several renditions of lab activities. If working with a community partner, the choice of organization and nature of the project is paramount to the success of the course. The community partner has to understand the limitations posed by working with undergraduates with limited analytical experience and the project has to be narrowed down to where it can be reasonably completed over the time frame of the course with the available

Table 5 Challenges and successes encountered in implementing active learning laboratory activities

Challenges

Choice of community partners

Common understanding with community partner of limitations imposed by working with undergraduates

Logistics of field trip

Students find it very hard to navigate scholarly literature

Lots of guidance needed to interpret written procedures and translate into experimental steps

Limited access to instrumentation

Difficult for one instructor to provide effective guidance - labs can be chaotic!

Students find scholarly articles hard to read

Difficulties with identifying materials and suppliers

Difficulties with calculations

Challenges in operating the instrumentation

Lab reports are involved

Successes

Students gain independence in lab

Students develop a completely new perspective on what it takes to implement an analysis

Samples are not from the stockroom!

Students gain knowledge of costs and budget

Students develop a deeper understanding of how to operate the instrumentation

Students gain awareness of the role of analytical chemistry in solving problems of interest to local community

Sense of worth and pride in sharing knowledge with community partner

Field trip is the highlight of the term!

Working with community partner is rewarding

Increased awareness of the role that federal and state organizations have on preserving surrounding areas

Increased self-esteem

Increased understanding of calculations involved in experiments

Opportunity to experience group work in light of future employment instrumentation. Students struggle researching and interpreting the literature, particularly translating written procedures into experimental steps. Labs can be chaotic and it is very challenging for one instructor to provide adequate guidance even with a class of 20 students or less. However, by the end of the term, students display much more independence and self-confidence. They gain a completely different perspective on what it takes to implement an analysis and develop a deeper understanding of the procedures and instrumentation used. The group work and divided responsibilities along with interacting with the community partner also provide valuable experience for future employment.

\section{Conclusion}

The implementation of an active learning analytical laboratory curriculum based on open-ended projects poses numerous challenges and careful attention must be given to the choice of project. Much time and effort is required of the instructor and students to successfully complete the analyses but the payoff is worth the struggle. Students gain hands-on knowledge, sharpen critical thinking skills, and improve lab skills while gaining self-confidence and independence.

Acknowledgments The author would like to thank Thomas Wenzel and Bates College for many valuable discussions. This work was supported in part by National Science Foundation grant DUE 1118600.

\section{References}

1. Handelsman J, Brown Q. A call to action: incorporating active STEM learning strategies into K-12 and higher education. August 17, 2016. https://www.whitehouse.gov/blog/2016/08/17/callaction-incorporating-active-stem-learning-strategies-k-12-andhigher-education. Accessed 9 May 2016.

2. Sandi-Urena S, Cooper M, Stevens R. Effect of cooperative problem-based lab instruction on metacognition and problemsolving skills. J Chem Educ. 2012;89:700-6.

3. Advisory Committee to the National Science Foundation Directorate for Education and Human Resources. Shaping the future: new expectations for undergraduate education in science, mathematics, engineering, and technology. Arlington, VA: National Science Foundation; 1996.

4. Kuwana T. Curricular developments in the analytical sciences. Washington, DC: National Science Foundation; 1998.

5. Wenzel TJ. Active learning materials for equilibrium chemistry and separation science. Anal Bioanal Chem. 2011;400:637-40.

6. The coalition for reform of undergraduate STEM education. In: Fry $\mathrm{CL}$, editor. Achieving systematic change: a source book for advancing and funding undergraduate STEM education. Washington, D.C.: The Association of American Colleges and Universities; 2014.

7. Kober N. Reaching students: what research says about effective instruction in undergraduate science and engineering. Washington, D.C.: The National Academies Press; 2015.

8. Graham M. Increasing persistence of college students in STEM. Science. 2013;341:1455-6. 
9. Freeman S, Eddy SL, McDonough M, Smith MK, Okoroafor N, Jordt $\mathrm{H}$, et al. Active learning increases student performance in science, engineering, and mathematics. Proc Natl Acad Sci. 2014;111:8410-5.

10. American Association of American Colleges \& Universities 2015 survey of employers and college students. http://www.aacu. org/sites/default/files/files/LEAP/2015employerstudentsurvey.pdf. Accessed 9 Aug 2016.
11. Analytical Sciences Digital Library. http://home.asdlib.org/. Accessed on 9 Aug 2016.

12. ASDL active learning curriculum materials, http://community. asdlib.org/activelearningmaterials/. Accessed 9 Aug 2016.

13. Harvey D. Analytical chemistry 2.0 online textbook. http://community.asdlib.org/activelearningmaterials/analyticalchemistry-2-0-online-textbook/. Accessed 9 Aug 2016. 